\title{
Simulação matemática de sintetização de um sinal sonoro obtido através de um violão Gianini
}

\section{Mathematical simulation of synthesizing a beep obtained through a Gianini guitar}

1 Paulo Sérgio Teixeira paulost39@gmail.com

1 Anderson Virgilio de Queiroz

1 Márcio Teodoro Fernandes

2 Alexandre José da Silva

2 José Flávio Silveira Feiteira

1 Doutorando em Engenharia Metalúrgica e Materiais - UFF.

2 Doutor em Engenharia Mecânica - UFF.

\section{Resumo}

A sintetização de sinais sonoros se torna cada vez mais um recurso utilizado no meio musical através de modelos matemáticos com o objetivo de prever o comportamento com relação às frequências geradas e produzir maior variedade de sons sintetizados computacionalmente. Um sinal acústico pode ser sintetizado a partir da transformada de Fourier. Os sinais podem ser escritos por uma soma de senos e cossenos combinados com características das frequências naturais de vibração, amortecimento, amplitudes, além da variação do fator tempo, ainda considerando que tais sinais sejam defasados $\pi / 2$ radianos, conforme estudado. Este trabalho apresenta uma abordagem metodológica para obter sinais sintetizados a partir de parâmetros medidos de sinais gerados por instrumentos musicais, objetivo central deste estudo. Os sinais acústicos originais foram obtidos através de gravação e editados no software Cool Edit 2000 com extensão wave, em seguida, para simulação dos sinais, foi utilizado o software Matlab para aplicação da transformada rápida de Fourier, e assim, obtendo-se os parâmetros de amortecimento, amplitudes, frequências naturais e amortecidas, gerando os sinais reconstruídos. Os sinais sintetizados obtidos como resultados no domínio da frequência, em comparação aos sinais medidos, apresentaram boas relações proporcionais de amplitude, mas com valores menores de amplitudes nos vários harmônicos.

\section{Palavras-chave:}

Sintetização. Sinais sonoros. Fourier. Ruído branco.

\begin{abstract}
The synthesis of sound signals is increasingly used in the musical environment through mathematical models to predict the behavior with respect to the generated frequencies and to produce a greater variety of synthesized sounds. An acoustic signal can be synthesized from the Fast Fourier Transform. The signals can be written by a sum of sines and cosines combined with characteristics of the natural frequencies of vibration, damping, amplitudes, in addition to the variation of the time factor, even considering that such signals are outdated $\pi / 2$ radians as studied (. This work presents a methodological approach to obtain signals synthesized from measured parameters of signals generated by musical instruments, the main objective of this study. The original acoustic signals were obtained through recording and edited in the software Cool Edit 2000 with wave extension, then for the simulation of the signals the Matlab software was used to apply the Fast Fourier Transform, thus obtaining the parameters of damping, amplitudes, natural and damped frequencies generating the reconstructed signals. The synthesized signals obtained as results in the frequency domain compared to the measured signals showed good proportional amplitude ratios but with lower amplitude values in the various harmonics.
\end{abstract}

\section{Keywords:}

Synthesizing. Sound signals. Fourier. White noise.

\section{Como você deve citar?}

TEIXEIRA, Paulo Sérgio et al.. Simulação matemática de sintetização de um sinal sonoro obtido através de um violão Gianini. Cadernos UniFOA, Volta Redonda, n. 39, p. 41-51, abril 2019. 


\section{INTRODUÇÃO}

Segundo Ynoguti (1995), existem várias razões para que, cada vez mais, músicos se utilizem da sintetização de sons ou sinais sonoros para o seu trabalho. Uma delas é a maior variedade de sons gerados pelo computador, que não são limitados àqueles disponíveis pelos instrumentos já existentes: pode-se gerar uma infinidade de novos sons através de processamentos digitais. Outra razão é a precisão com que o computador trata os sinais: é capaz de analisar e processar a forma de onda do som até o nível da amostra individual.

Um sinal acústico pode ser sintetizado a partir da transformada de Fourier. Os sinais podem ser escritos por uma soma de senos e cossenos combinados com características das frequências naturais de vibração, amortecimento, amplitudes, além da variação do fator tempo. Essas características podem ser obtidas através da análise das características do sinal no domínio do tempo e frequência com o objetivo de se separar as frequências excitadas para posterior filtragem e, por conseguinte, determinar as características de amortecimento.

A Série de Fourier, desenvolvida por Jean Baptiste Joseph Fourier (1768-1830), matemático da corte de Napoleão, que teve seu nome imortalizado pelas séries trigonométricas introduzidas em 1807 e que, até hoje, são admiradas por matemáticos, físicos, estatísticos e engenheiros, são valiosas para quem precisa descrever uma função relativamente complexa, de uma forma simples de se visualizar e de se manipular. Ele mostrou que qualquer função, por mais complicada que seja, pode ser expressa como uma soma de senos e cossenos (BASSALO, 2009).

Há muito tempo, são estudadas as funções seno e cosseno, aprendendo-se que suas amplitudes originais são unitárias e que ambas estão defasadas entre si de $\pi / 2$ radianos, conforme Equação 1 (FEITEIRA, FONSECA e CAMPOS, 2013).

$$
\cos (x)=\operatorname{sen}\left(x+\frac{\pi}{2}\right)
$$

Onde x é o valor de um ângulo em radianos.

A série de Fourier é a representação de uma função periódica como uma soma de funções periódicas da forma de harmônicas de $\mathrm{e}^{\mathrm{i} x}$. De acordo com a fórmula de Euler, as séries podem ser expressas equivalentemente em termos de funções seno e cosseno (SANDIFER, E., 2007).

Fourier descobriu que funções periódicas de período fundamental T podem ser expressas como uma série infinita de funções trigonométricas na forma, conforme Equação 2 e, em sua forma mais compacta, na Equação 3 :

$$
\begin{gathered}
\frac{a_{0}}{2}+a_{1} \cos (x)+b_{1} \operatorname{sen}(x)+a_{2} \cos (2 x)+b_{2} \operatorname{sen}(2 x)+\ldots+a_{n} \cos (n x)+b_{n} \operatorname{sen}(n x) \\
\frac{a_{0}}{2}+\sum_{n=1}^{\infty}\left(a_{n} \cos (n x)+b_{n} \operatorname{sen}(n x)\right)
\end{gathered}
$$

Onde x é o valor de um ângulo em radianos, a é amplitude, n número de termos da equação.

O objetivo geral do presente trabalho é a sintetização de sinais a partir da transformada de Fourier. $\mathrm{E}$, para isso, foi realizado estudo de características relevantes à resposta dada por um instrumento de corda. 


\subsection{Transformada de Fourier}

Após a teoria de Fourier, que se propunha decompor funções periódicas em somas de funções senoidais, surgiu a transformada de Fourier e depois a transformada rápida de Fourier (FFT - Fast Fourier Transform).

Uma função pode ser convertida do domínio do tempo para o da frequência através de um operador matemático chamado genericamente de transformada integral. Transformada de Fourier decompõe uma função na soma de um (potencialmente infinito) número de componentes senoidais, produzindo um espectro de frequências. A transformada inversa correspondente converte esse espectro de volta para o domínio do tempo, ou seja, para a função original.

Ao aplicar-se a transformada de Fourier, passa-se do domínio do tempo para o domínio da frequência e a informação a respeito de deslocamentos de fase do sinal em função da frequência desaparece.

Em acústica e música, a fundamental é a mais baixa frequência e a de maior amplitude das componentes da série harmônica de um som. Tecnicamente, a fundamental corresponde ao primeiro harmônico. Na realidade, o som é composto de várias frequências, mas as que se destacam são as que entram em ressonância, fenômeno que acontece quando a vibração atinge a chamada frequência natural de vibração do meio. A frequência fundamental ou a primeira que entra em ressonância é responsável pela percepção da altura de uma nota, enquanto que as demais, chamadas de harmônicos, participam da composição da forma de onda do som.

\subsection{Análise Sonora de Instrumentos Musicais}

Para Lazzarini (1998), o som é uma qualidade perceptiva que é o resultado da percepção de distúrbios das moléculas de um meio em certo espaço de tempo. Esses distúrbios, por sua vez, apresentam-se em forma de ondas em sua propagação pelo meio.

O som é produzido ao criarmos algum tipo de mecanismo que altere de forma periódica a pressão do ar em nossa volta (WUENSCHE, 2009).

Segundo Lacerda (1996), o som pode ser representado por uma soma de diversas ondas individuais chamadas de componentes de Fourier e cada uma corresponde a uma determinada frequência múltipla da componente inicial. Essas componentes formam uma série conhecida como série harmônica, onde o harmônico de ordem zero é chamado de frequência natural ou fundamental, o segundo é denominado harmônico de primeira ordem ou primeiro harmônico, o terceiro é chamado segundo harmônico e assim sucessivamente.

As amplitudes e o tempo de duração de cada um dos harmônicos presentes no som resultante podem variar independentemente. A combinação dessas duas propriedades tem o nome de timbre.

A síntese sonora de timbres de instrumentos musicais depende, basicamente, do conjunto de harmônicos utilizados (DODGE; JERSE, 1997).

Para analisar os sinais acústicos de instrumentos, nesse caso, o violão, é necessário considerá-lo com um sistema vibrante. 
Os sistemas de vibração podem vibrar em vários modos diferentes. Cada modo é relacionado a uma frequência diferente e, portanto, um modo de vibração pode ser excitado individualmente por algum tipo de perturbação relacionado à certa frequência.

A descrição desse movimento vibracional é bastante difícil, pois é necessário saber a amplitude (ou intensidade) e a frequência de cada modo de vibração perturbado.

O espectro de uma vibração indica quais frequências estão sendo excitadas e com que intensidade. Essa análise espectral, também conhecida como análise no domínio da frequência, é obtida através da análise de Fourier do sinal sonoro, especificamente pela aplicação da transformada de Fourier ao sinal temporal representativo da vibração estudada.

\subsection{Amortecimento de Sinais}

Segundo Cossolino e Pereira (2010), o amortecimento, ou atrito interno, é uma das propriedades mais sensíveis de materiais e estruturas, tanto em escala macro quanto microscópica. É o fenômeno pelo qual a energia mecânica de um sistema é dissipada (principalmente pela geração de calor e/ou energia). 0 amortecimento determina a amplitude de vibração na ressonância e o tempo de persistência da vibração depois de cessada a excitação. Em sistemas dinâmicos, o amortecimento é fenômeno pelo qual a energia mecânica é dissipada (DE SILVA, 2007).

O amortecimento de um sistema ou material pode ser classificado de três formas principais: interno, estrutural e fluídico. 0 interno está associado aos defeitos na microestrutura, granularidade, impurezas do material e a efeitos termo elásticos causados por gradientes locais de temperatura. Já o estrutural, está associado a perdas de energia por atrito em juntas, parafusos e articulações semirrígidas. Por último, o fluídico ocorre por resistência ao arraste em meio fluídico, por exemplo, a conversão de energia cinética de um pêndulo em energia térmica para o ar.

Existem diversos métodos para determinação do amortecimento, os quais podem ser obtidos basicamente por dois caminhos: mediante a duração da resposta do sistema a uma excitação transitória (exemplo: método do decremento logarítmico) e em função da resposta do sistema em função da frequência (exemplo: método da largura de meia banda de potência). 0 método do decremento logarítmico calcula o amortecimento a partir da atenuação da resposta acústica do material ou estrutura após uma excitação por impulso. A importância de se calcular o amortecimento está no fato de que esse parâmetro pode indicar como a energia de vibração é absorvida pelo meio material onde se propaga. E esses valores podem também ser utilizados na sintetização de sinais através das Equações 4 e 5. O decremento logarítmico é calculado a partir de um simples impulso provocado no sistema (em vibração livre) através da razão entre duas amplitudes sucessivas do sinal. 0 termo decremento logarítmico refere-se à taxa de redução logarítmica, relacionada com a redução do movimento após o impulso, pois a energia é transferida para outras partes do sistema ou é absorvida pelo próprio elemento.

Quando um sistema oscilatório com um grau de liberdade com amortecimento viscoso é excitado por um impulso, sua resposta vem na forma de decaimento no tempo conforme figura 1. 
Figura 1 - Resposta ao Impulso

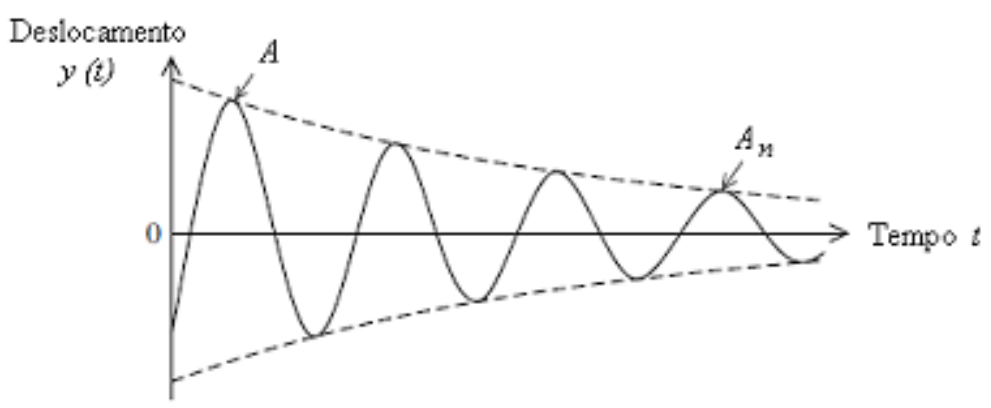

Fonte: Cossolino e Pereira, 2010.

$$
y(t)=y e^{-\varsigma w_{0} t} \operatorname{sen}\left(w_{d} t\right)
$$

Onde y é a amplitude, $\zeta$ é o amortecimento, $\mathrm{w}_{0}$ é a frequência natural e $\mathrm{w}_{d}$ frequência natural amortecida.

Para isso, define-se $\zeta$ :

$$
\zeta=\frac{1}{2 \pi} \cdot \frac{1}{n} \ln \left(\frac{A_{1}}{A_{n}}\right)=\frac{\delta}{2 \pi} \quad \text { para } \zeta<0,1
$$

onde $\mathrm{n}$ é números de amplitudes, A1 é a amplitude do primeiro ciclo é , An é a amplitude de $\mathrm{n}$ ciclos, $\delta$ é o decremento logarítmico.

Após n períodos, o amortecimento pode ser calculado em função da razão de outra amplitude.

A Equação 5 foi usada para análise de sinais para determinação do amortecimento dos sinais captados de cordas. 
Para a solução $0 \leq \zeta<1$, o sistema subamortecido utiliza a Equação 6:

$$
x(t)=A_{0} e^{-\zeta \omega_{0} t} \cos \left(\omega_{d} t+\varphi\right)
$$

Onde $A_{0}$ é a amplitude inicial de vibração, $\varphi$ é a fase inicial de vibração e $\omega_{d}$ é chamada de frequência natural amortecida e é descrita na Equação 7.

$$
\omega_{d}=\omega_{0} \sqrt{1-\zeta^{2}}
$$

A Equação 7 é válida para um modelo conhecido como sistema linear amortecido com um grau de liberdade. Dessa forma, assume-se que a ressonância de materiais pode ser vista como uma associação de vários sistemas de um grau de liberdade. 0 modelo de vibração pode ser observado na Equação 8:

$$
x(t)=\left\lfloor\sum_{i=1}^{N} A_{i} e^{-\zeta_{i} \omega_{n i} t} \cos \left(\omega_{d i}+\varphi_{i}\right)\right\rfloor+R_{w n}
$$

Em que $A_{i}, \zeta_{i}, \omega_{n i}, \omega_{d i}, \varphi_{i}$, são, respectivamente, amplitude inicial, amortecimento, frequência natural de vibração, frequência natural amortecida e fase inicial do i-ésimo modo de vibração. 0 termo $R_{\omega n}$ é um ruído branco descorrelacionado do sinal.

\section{MATERIAIS E MÉTODOS}

Inicialmente, busca-se caracterizar qualidades relacionadas ao amortecimento das vibrações a partir de respostas sonoras gravadas e analisadas nos domínios de tempo e frequência. Para tal, foram gravadas respostas sonoras do violão da marca Gianini, sete cordas, tampo de cedro e corda Gianini ginga.

\subsection{Obtenção de Sinais a Serem Sintetizados}

O método consiste em produzir respostas sonoras a partir de dedilhamento de notas musicais com base na escala musical para instrumentos de afinação temperada. Para isso, cada nota foi tocada separadamente, abafando-se as demais para não haver interferência de vibração.

Para captação da resposta sonora, foi usado microfone e, para a gravação dos sinais, foi usado o software Cool Edit 2000.

A primeira etapa depois da gravação é a transferência dos sinais para serem lidos no software Matlab, gerando-se gráficos de amplitude versus tempo. Em seguida, é feita a conversão para o domínio da frequência através da transformada rápida de Fourier (FFT), separando-se em uma primeira análise a frequência fundamental. A terceira etapa consiste em filtrar essa frequência pelo método de largura de banda, gerando um sinal no domínio do tempo. Logo depois, se faz necessário a aplicação da FFT para 
verificar a filtragem do sinal. Para tal, deverá aparecer no gráfico amplitude versus frequência, somente um pico com grande amplitude, o da frequência filtrada. Por último, deve-se retornar o sinal para o domínio do tempo através da transformada inversa de Fourier, separando-se os valores de primeiro pico de maior amplitude e o décimo para, posteriormente, serem usados no cálculo do amortecimento dessa frequência através do método do decremento logarítmico (TEIXEIRA, SILVA; FEITEIRA, 2014).

E, assim, utilizando-se da transformada de Fourier juntamente com as equações de Euler, é feita a reprodução ou sintetização de sinal escrito por uma soma de senos ou cossenos, nesse caso, cossenos, combinados com características das frequências naturais de vibração, amortecimento, amplitudes, além da variação do fator tempo (Equações 6 e 7 ).

Para obtenção desses dados a partir de um sinal gravado, se faz necessário repetir o procedimento adotado para obtenção dos valores de amortecimento, separando-se cada frequência natural do sinal juntamente com a amplitude inicial, taxa de amortecimento, frequência natural amortecida, ângulo de defasagem do sinal. Todos esses valores se repetem para cada frequência natural ou componente harmônica. Isso é repetido várias vezes, fazendo-se um somatório que será o resultado final do sinal sintetizado (Equação 8).

\subsection{Sintetização de Sinais}

O sinal utilizado para a demonstração da sintetização é a nota $\mathrm{La}_{4}$ ou $\mathrm{A}_{4}$ do violão Gianini que tem como frequência fundamental 440 hertz. Além dessa, no novo sinal, deverá conter a representação de mais oito harmônicos.

Para compor o sinal, inicialmente, deve-se calcular o amortecimento da frequência harmônica que compõe o sinal. E, para isso, deverá ser extraído a amplitude inicial e a amplitude após dez ciclos. Depois se calcula a frequência natural amortecida, de acordo com Equação 7 que, juntamente com a amplitude inicial e a frequência natural excitada, monta-se cada componente do sinal através da Equação 6. Somando todas as componentes (Equação 8), tem-se a representação do sinal.

Quanto mais componentes harmônicas forem somadas, mesmo aquelas com intensidades iniciais baixas, mais completo será o sinal e mais próximo do real será.

Durante a captação do sinal que será reproduzido, pode surgir um componente do sinal chamado de ruído branco $\left(R_{w n}\right)$, que deverá ser analisado para se verificar se este fará ou não parte do novo sinal. Em alguns casos, esses ruídos são indesejáveis e difíceis de serem controlados e podem ser suprimidos durante a sintetização.

A seguir foi desenvolvida, no Matlab, uma sequência de rotinas a partir da equação 8, que produz a sintetização desses sinais.

Na composição do novo sinal, não foram adicionadas as frequências ruidosas (ruídos brancos) que aparecem nos sinais originais.

\section{$3 \quad$ RESULTADOS E DISCUSSÕES}

Na Tabela 1, estão os valores medidos de frequências naturais ou harmônicos, as frequências de corte utilizadas para filtrar o sinal, as amplitudes iniciais e após dez ciclos, assim como os valores calculados de amortecimento. 
Tabela 1 - Dados para cálculo das taxas de amortecimento da frequência fundamental e harmônicos da nota A4 do violão Gianini para sintetização.

\begin{tabular}{cccccc}
\hline $\begin{array}{c}\text { Frequência } \\
\text { (hertz) }\end{array}$ & \multicolumn{2}{c}{ Frequência de corte } & Amplitude & Amplitude & Amortecimento \\
(hertz) & Superior & 1 & & \\
& Inferior & ciclos $)$ & \\
\hline 439,6 & 400 & 460 & 0,3174 & 0,293 & $1,273084 \mathrm{E}-03$ \\
\hline 876 & 865 & 890 & 0,1123 & 0,1088 & $5,03925 \mathrm{E}-04$ \\
\hline 1319 & 1313 & 1328 & 0,1095 & 0,109 & $7,28399 \mathrm{E}-05$ \\
\hline 1758 & 1740 & 1770 & 0,02083 & 0,02075 & $6,1243 \mathrm{E}-05$ \\
\hline 2175 & 2214 & 2199 & 0,01328 & 0,01321 & $8,41138 \mathrm{E}-05$ \\
\hline 2638 & 2632 & 2646 & 0,03563 & 0,03505 & $2,61211 \mathrm{E}-04$ \\
\hline 3079 & 3072 & 3090 & 0,01374 & 0,0137 & $4,64009 \mathrm{E}-05$ \\
\hline 3520 & 3505 & 3535 & 0,02411 & 0,02383 & $1,85915 \mathrm{E}-04$ \\
\hline 3963 & 3960 & 3975 & 0,009202 & 0,009182 & $3,4629 \mathrm{E}-05$ \\
\hline
\end{tabular}

Fonte: próprios autores, 2019.

Nas figuras 2 e 3, respectivamente, estão os sinais gravados no domínio do tempo e, após transformada rápida de Fourier (FFT), no domínio da frequência.

Figura 2 - Nota Lá $\left(\mathrm{A}_{4}\right)$ do violão Gianini no domínio do tempo.

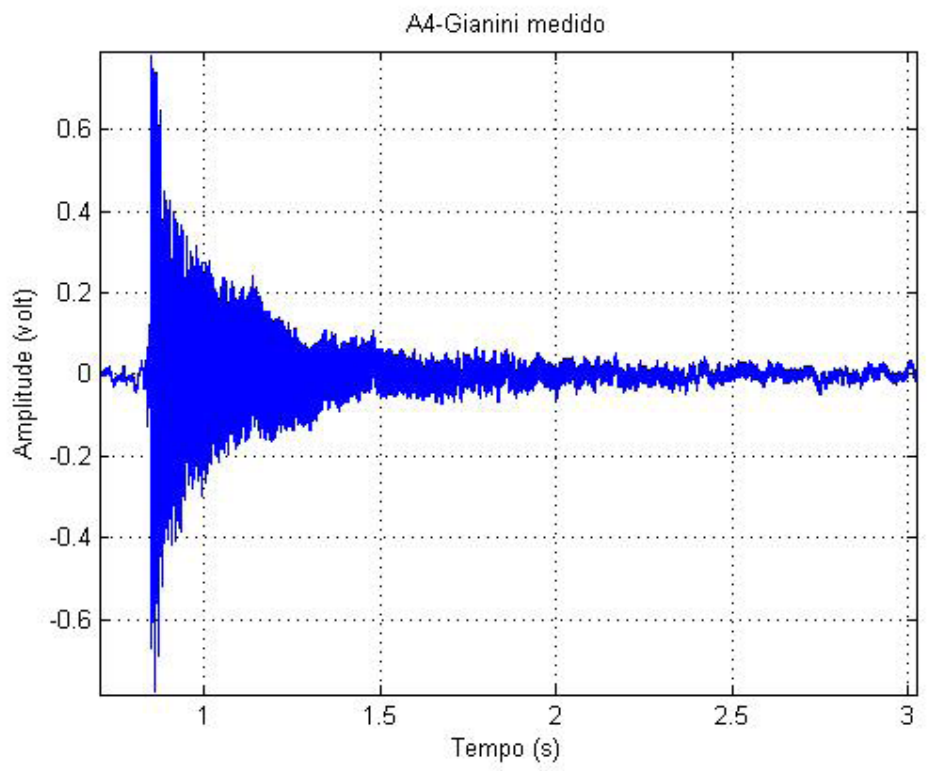

Fonte: próprios autores, 2019. 
Figura 3 - Nota Lá $\left(\mathrm{A}_{4}\right)$ do violão Gianini no domínio da frequência.

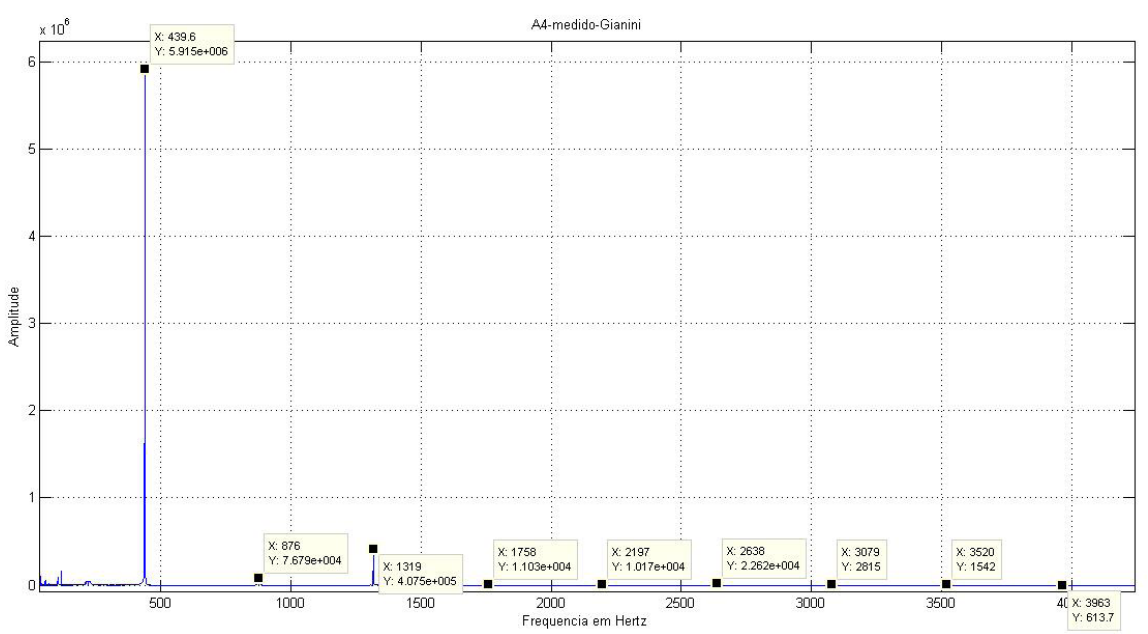

Fonte: próprios autores, 2019.

Nas Figuras 4 e 5, está demonstrado o resultado do sinal sintetizado no domínio do tempo, mostrando uma densidade espectral parecida com a do sinal medido e desaparecendo alguns picos provenientes, provavelmente de ruídos brancos e, na figura 5 , os resultados no domínio da frequência, onde estão demonstrados a frequência fundamental e mais oito harmônicos.

Figura 4 - Nota A4 do violão Gianini sintetizada no domínio do tempo.

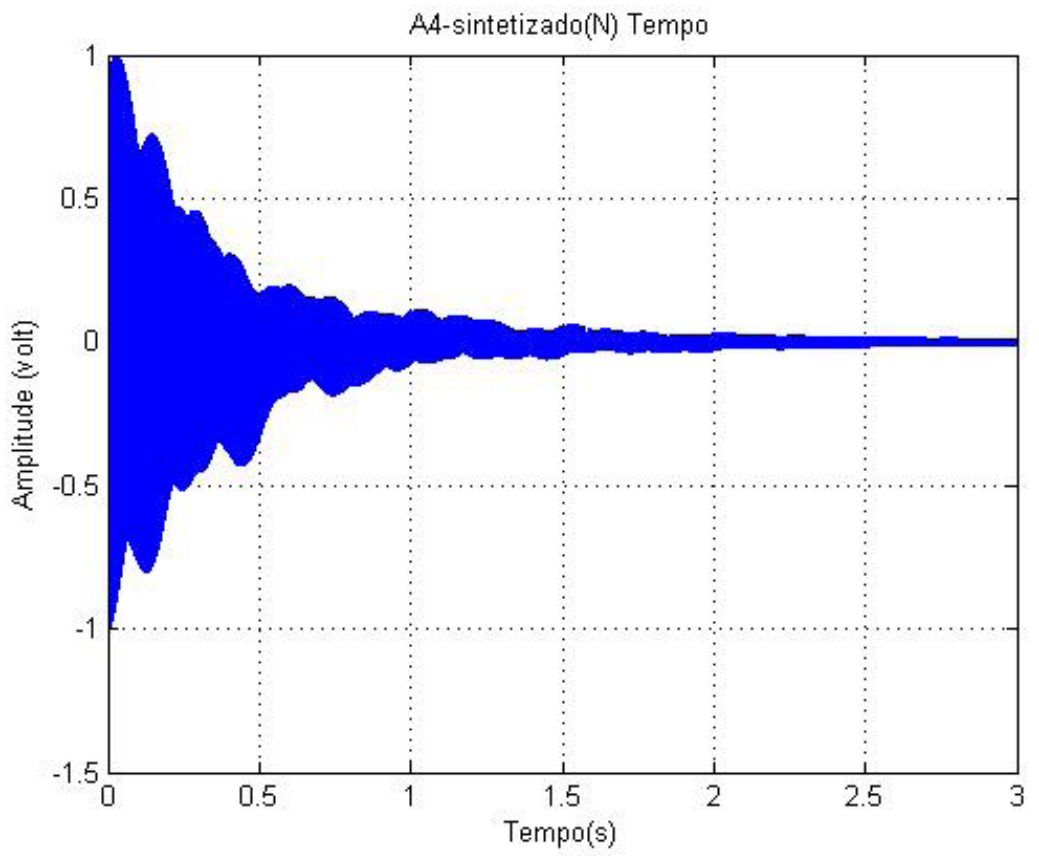

Fonte: próprios autores, 2019. 
Figura 5 - Nota A4 do violão Gianini sintetizada no domínio da frequência.

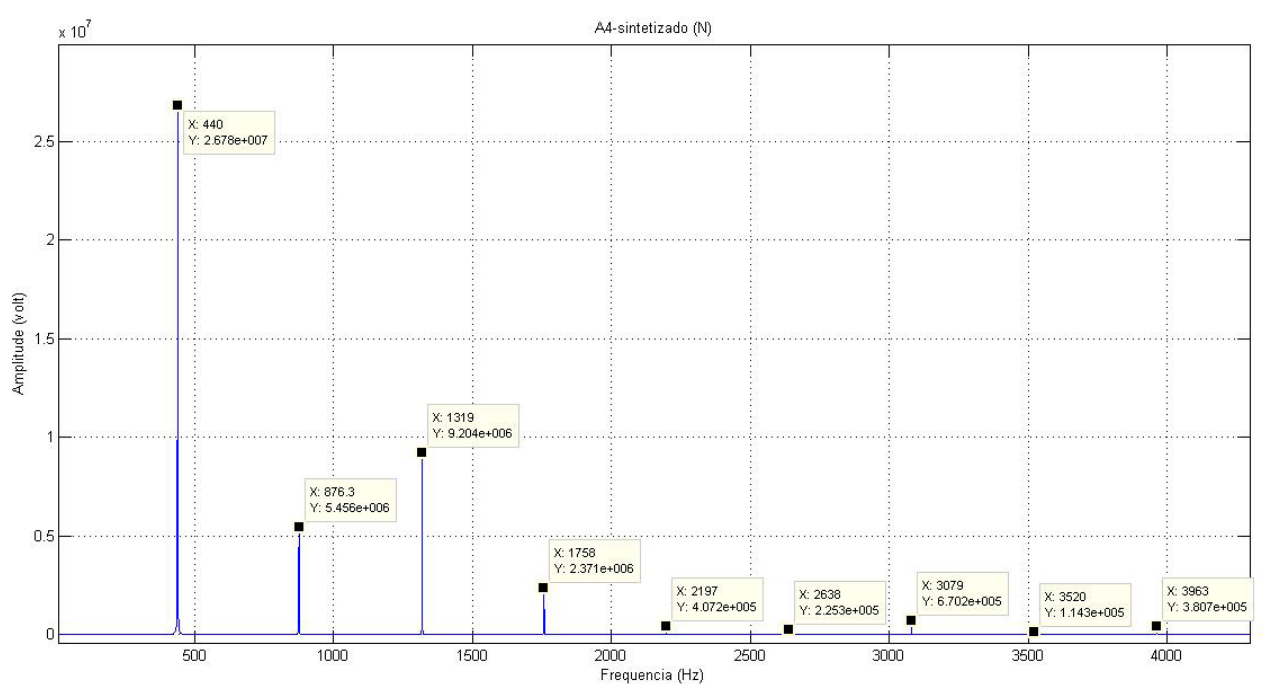

Fonte: próprios autores, 2019.

Os sinais sintetizados no domínio da frequência, em comparação aos sinais medidos, mostram relações proporcionais de amplitude, mas com valores menores de amplitudes nos vários harmônicos. Isso pode se dar pelo fato de que o sinal perde energia quando é filtrado e que algumas frequências de pequenas amplitudes são suprimidas. São os chamados ruídos brancos que poderão, ou não, fazer parte do sinal sintetizado.

Os resultados da sintetização apresentaram uma boa correlação com o medido, tanto no domínio do tempo, quanto no domínio da frequência. Mas um dos principais parâmetros usados deve ser muito bem controlado, o amortecimento. Foi percebido durante a pesquisa que ele influencia diretamente na amplitude de frequência, tanto que é um fator que pode sofrer ajustes para melhorar o resultado do sinal sintetizado. 0 amortecimento por ser calculado através de uma média logarítmica de dez amplitudes sucessivas, mas nem sempre reproduz a taxa de amortecimento do sinal como todo.

\section{CONCLUSÃO}

O método de sintetização apresentado neste trabalho se mostra bastante eficiente no que diz respeito à apuração de sinais acústicos, com relação a ruídos indesejáveis (ruído branco), que, muitas vezes, são difíceis de serem controlados no processo de medição. Dessa forma, o método apresentado é uma ferramenta útil para a melhoria do sinal.

Uma das limitações desse método é que a soma de senoides de todas as frequências, incluindo as de pequenas amplitudes, podendo exigir um esforço computacional grande.

Quanto ao ruído branco, nem sempre há a necessidade de eliminá-lo, pois ele faz parte da composição sonora de vários sinais acústicos e isso poderia representar, em uma análise mais subjetiva, a perda de qualidade do som. 


\section{REFERÊNCIAS}

BASSALO, J.M. As Séries de Fourier. Seara da Ciência. Disponível em: http://www.seara.ufc.br/tintim/ matematica/fourier/fourier1.htm. Acesso em: 05 ago. 2015.

COSSOLINO L.C.; PEREIRA A.H.A. Amortecimento: classificação e métodos de determinação (informativo técnico científico) - Universidade de São Carlos, 2010.

DE SILVA, C. Vibration Damping, control and design. CRC Press, 2007.

DODGE, C.; JERSE, T. A. - Computer music synthesis, composition, and performance. 2. ed. New York: Charmer Books, 1997.

FEITEIRA, J. F.; FONSECA, G. S.; CAMPOS, M. F. “ Série e Transformada de Fourier na Análise Cristalográfica por Difração de Raio X". $7^{\circ}$ Congresso Brasileiro de Engenharia de Fabricação, Penedo, 2013.

LACERDA, O.. Compêndio de teoria elementar da música. 9. ed. Ricordi Brasileira S.A., 1996.

LAZZARINI, Victor E. P.. Elementos de acústica. Music Department - National University of Ireland, Maynooth, 1998.

SANDIFER, E. Some Facets of Euler's Work on Series, Studies in the History and Philosophy of Mathematics. Volume 5, Pages 279-302C, 2007.

TEIXEIRA, P. S.; SILVA, A. J.; FEITEIRA, J. F.. Avaliação e comparação de características de amortecimento de sinais gerados de diferentes violões. Caderno UniFOA, 2014.

YNOGUTI, Carlos Alberto. Síntese de sons musicais baseada na transformada Karhunem-Loève. São Carlos. Dissertação de Mestrado, Escola de Engenharia de São Carlos, Universidade de São Paulo. 1995.

WUENSCHE, Carlos A. Física da Música . inpe/mct - Divisão de Astrofísica, 2009. 